\title{
Association between gait speed and bone strength in community-dwelling postmenopausal Japanese women
}

\author{
Takahiko Sakazaki • Teruhiko Koike • \\ Yuji Yanagimoto $\cdot$ Yoshiharu Oshida
}

Received: 5 November 2011/Accepted: 10 January 2012/Published online: 28 January 2012

(C) The Japanese Society for Hygiene 2012

\begin{abstract}
Objectives It is unclear whether gait speed can be used to predict bone status or to diagnose osteopenia or osteoporosis. The aim of our study was to determine whether the measurement of physical performance predicts bone strength in community-dwelling postmenopausal Japanese women.

Methods We measured speed of sound (SOS), weight bearing index (WBI), maximum or usual gait speed, step length of each gait, single leg balance time with or without eyes closed, and grip strength among 1,061 postmenopausal women (mean age \pm standard deviation $68 \pm 8$ years).

Results After adjustments for age and body mass index, SOS was associated with maximum or usual gait speed and with step length during maximum speed gait, but not with WBI, grip strength, single-leg balance time with or without eyes closed, or step-length time during usual gait speed. When subjects were divided into quartile groups based on WBI (a marker of lower extremity strength), significant associations between usual or maximum gait speed and SOS were found only in the lowest and second lowest groups (first and second quartiles). Finally, analysis of sensitivity and specificity at various cut-off points of
\end{abstract}

T. Sakazaki $(\square)$

Department of Sports Medicine, Graduate School of Medicine,

Nagoya University, Furo-cho, Chikusa-ku, Nagoya,

Aichi 464-8601, Japan

e-mail: sakazaki.takahiko@g.nagoya-u.jp

T. Koike $\cdot$ Y. Oshida

Research Center of Health, Physical Fitness and Sports,

Nagoya University, Nagoya, Japan

Y. Yanagimoto

Department of Nursing, Faculty of Health Science,

Kobe Tokiwa University, Kobe, Japan maximum gait speed to predict decreased SOS showed relatively high specificity compared with the corresponding sensitivity among those with low gait speed.

Conclusions Maximum gait speed together with WBI may be a useful and specific test to predict bone status in postmenopausal older women.

Keywords Weight bearing index - Speed of sound - Gait speed $\cdot$ Postmenopausal women $\cdot$ Physical function

\section{Introduction}

Life expectancy in developed countries was prolonged by approximately 30 years during the twentieth century. The resulting increase in the number of elderly in the general population represents a challenge in terms of the ability of society to cope with the decline in physical and/or mental function of frail elderly individuals. Frailty is a geriatric syndrome resulting from age-related cumulative declines across multiple physiologic systems [1]. Sarcopenia (agerelated decrease of muscle mass) [2] and osteoporosis [3] are classic geriatric syndromes that directly affect the activities of elderly persons. Sarcopenia, a risk factor for falls, and osteopenia or osteoporosis, a risk factor for bone fracture, increase the morbidity and mortality among elderly people.

The evaluation of physical performance can be crucial for the diagnosis of frailty. Physical performance measures have been shown to predict adverse health-related events [4], onset of difficulties with activities of daily living [5], or mortality [6] in older community-dwelling populations. Gait speed is the most frequently used physical performance measurement, and an association between gait speed and survival in older adults has been reported [7]. 
Likewise, slow walking speed in older people has been shown to be strongly associated with an increased risk of cardiovascular mortality [8]. An algorithm developed by the European Working Group on Sarcopenia in Older People (EWGSOP) [9] to identify sarcopenia proposed gait-speed measurement as the first step of the algorithm.

It is unclear whether gait speed can be used to predict bone status or to diagnose osteopenia or osteoporosis. Bone mineral density (BMD) is currently used to diagnose osteoporosis, and its decrease is the major risk factor for bone fracture. We measured heel bone density by ultrasound, as this measurement has been shown to predict the risk of hip fracture as efficiently as BMD measurement $[10,11]$. We hypothesized that subjects with weak bone status could be identified based on physical performance measures, including gait speed. Our results indicate that the combination of lower extremity strength and gait speed may provide a tool for screening subjects suffering from both bone and muscle functional decline.

\section{Subjects and methods}

\section{Study population}

This study was conducted from 2002 to 2007 and was supported in part by Wellness Core 21, a nonprofit organization with it headquarters in Kobe, Japan. Of the initial 1,946 subjects, 885 were excluded from the analysis due to a lack of data. The final study population included 1,061 community-dwelling postmenopausal Japanese women aged 55-90 years [mean \pm standard deviation (SD) $68 \pm 8$ years]. The subjects were grouped into four age classes, namely 55-64 years (372 subjects), 65-74 years (466), 75-84 years (210), and 85-90 years (13). They were contacted through various publicity efforts 2 months before the start of the study in Kobe City, Nishinomiya City, Kami-town, and Yashiro Town, and voluntarily participated in the study. The protocol and consent form were approved by the ethics committee of the Wellness Core 21 nonprofit organization, and written, informed consent was given by all study participants. The protocol was approved by the ethics committee of the Research Center of Health, Physical Fitness and Sports, Nagoya University.

\section{Measures}

Body weight $(\mathrm{kg})$ was measured to the nearest $0.1 \mathrm{~kg}$ with a weight scale (Tanita, Tokyo, Japan), and height $(\mathrm{cm})$ was measured to the nearest $0.1 \mathrm{~cm}$ using a scale attached to the wall. The body mass index (BMI) is defined as the weight $(\mathrm{kg})$ divided by height $(\mathrm{m})$, squared. Bone quality was measured using an ultrasound bone-density measuring device (CM-100; Furuno Electric Co., Nagasaki, Japan). The coefficient of variance $(\mathrm{CV})$ was $1.6 \%$. The measurements were performed at the right heel, and speed of sound (SOS), which represents the velocity of ultrasound transmission through the bone (in this case, the calcaneus), was obtained $(\mathrm{m} / \mathrm{s})$ [12]. Maximum grip strength was measured using a handgrip dynamometer. Grip strength was measured two times on each side, and the higher values for each side were used to calculate the average. Single-leg stance balance was used to assess body balance. The length of time that the subject could stand on her right or left leg with or without her eyes closed and without hand support was measured with a stopwatch for up to $180 \mathrm{~s}$. Gait speed was measured using a $10-\mathrm{m}$ straight one-way path. To allow for acceleration, participants started to walk $1 \mathrm{~m}$ before the marker that was used to indicate when the time for the gait-speed measurement was started. Participants were instructed to stand with both feet touching the starting line and to begin walking from the standing position at their usual or maximum pace after a verbal command. No participants used a cane or a walker, but we did not check the use of orthosis. Mean step length was determined based on the length of the second through to the final recorded step because the first step length tends to be shorter than that of subsequent steps [13].

Knee extensor power was measured isometrically by a dynamometer (Yagami Co., Nagoya, Japan). Subjects were seated in a steel chair with their lower leg unsupported and knee and hip flexed to a $90^{\circ}$ angle [14]. Strength was measured as the force applied to the lower leg and measured one time each in both legs. Added value was used for weight bearing index (WBI), which was calculated as leg strength (kg)/body weight $(\mathrm{kg})$ [15].

\section{Statistical analysis}

Data were analyzed using the Statistical Package for Social Science for Windows ver. 17 (SPSS Japan, Tokyo, Japan). Means and SD were obtained for measured variables. A $p$ value of $<0.05$ was considered to be statistically significant. The association between SOS and variables in physical performance tests was examined using univariate and multiple linear regressions.

\section{Results}

Subject characteristics

The general characteristics of the participants are presented in Table 1. All subjects were community-dwelling postmenopausal women who volunteered to participate in the physical performance tests. About three-quarters of the 
Table 1 General characteristics of participants

\begin{tabular}{|c|c|c|c|c|c|}
\hline Characteristics & $\begin{array}{l}\text { All } \\
(n=1,061)\end{array}$ & $\begin{array}{l}55-64 \text { years } \\
(n=372)\end{array}$ & $\begin{array}{l}\text { 65-74 years } \\
(n=466)\end{array}$ & $\begin{array}{l}75-84 \text { years } \\
(n=210)\end{array}$ & $\begin{array}{l}85-90 \text { years } \\
(n=13)\end{array}$ \\
\hline Age (years) & $68(8)$ & $60(3)$ & $69(3)$ & $79(3)$ & $87(2)$ \\
\hline Height $(\mathrm{cm})$ & $151.2(6.1)$ & $153.8(5.4)$ & $151.0(5.4)$ & $147.0(5.9)$ & $143.7(6.3)$ \\
\hline Weight (kg) & $51.7(7.5)$ & $53.3(7.3)$ & $51.5(7.3)$ & $49.4(7.8)$ & $45.4(6.6)$ \\
\hline Body mass index $\left(\mathrm{kg} / \mathrm{m}^{2}\right)$ & $22.6(2.9)$ & $22.5(2.8)$ & $22.6(2.8)$ & $22.9(3.4)$ & $21.9(2.6)$ \\
\hline Speed of sound $(\mathrm{m} / \mathrm{s})$ & $1,489.7(24.0)$ & $1,498.7(25.0)$ & $1,489.0(21.0)$ & $1,476.9(21.9)$ & $1,464.9(11.8)$ \\
\hline Grip strength (kg) & $22.2(4.7)$ & $24.4(3.9)$ & $21.8(4.5)$ & $18.8(4.1)$ & $15.8(3.7)$ \\
\hline Single-leg balance with eyes closed (s) & $10.1(14.1)$ & $16.8(19.7)$ & $7.7(8.7)$ & $4.2(4.2)$ & $2.4(2.6)$ \\
\hline Single-leg balance without eyes closed (s) & $49.2(35.5)$ & $71.4(27.9)$ & $46.8(34.3)$ & $17.6(19.7)$ & $8.1(14.2)$ \\
\hline Maximum gait speed (m/min) & $104.2(22.9)$ & $117.5(17.2)$ & $103.9(19.9)$ & $84.1(19.2)$ & $55.9(16.4)$ \\
\hline Step length during maximum speed gait $(\mathrm{cm})$ & $71.9(12.0)$ & $77.4(9.5)$ & $72.4(10.8)$ & $62.8(12.0)$ & $47.9(11.7)$ \\
\hline Usual gait speed $(\mathrm{m} / \mathrm{min})$ & $82.7(18.3)$ & $91.5(13.7)$ & $83.8(17.1)$ & $66.7(15.1)$ & $48.7(15.0)$ \\
\hline Step length during usual speed gait $(\mathrm{cm})$ & $65.5(10.7)$ & $70.2(8.1)$ & $66.1(10.2)$ & $56.8(9.8)$ & $44.7(11.9)$ \\
\hline Weight bearing index & $0.73(0.25)$ & $0.82(0.24)$ & $0.73(0.26)$ & $0.59(0.19)$ & $0.43(0.07)$ \\
\hline
\end{tabular}

Values are given as the mean, with the standard deviation (SD) in parenthesis

participants were non-obese, $24.2 \%$ were overweight (BMI $25.0-29.9 \mathrm{~kg} / \mathrm{m}^{2}$ ), and $1.4 \%$ were obese $\left(\mathrm{BMI}>30 \mathrm{~kg} / \mathrm{m}^{2}\right)$.

Association between SOS and physical performance measures

Table 2 shows the association of SOS with other physical performance variables after adjustments for age and BMI. All of the variables were negatively associated with age (data not shown). Grip strength had a positive correlation with BMI, while single-leg balance, gait speed, and step length had a negative correlation with BMI (data not shown). SOS had a significant association with maximum or usual gait speed and with the step length of the maximum gait speed.

Association between gait speed and SOS in the quartile groups (based on WBI)

To analyze the association between gait speed and SOS in more detail, we divided subjects into quartiles based on WBI levels. A significant association between maximum gait speed and SOS was found in the each quartile, but following adjustments for age and BMI, the association remained significant only in the first or second quartile (Table 3 ). The average and range of the maximum gait speed in the first, second, third and fourth quartiles were $87.2 \pm 23.2$ and 22.4-140.8, $103.1 \pm 21.8$ and 44.4-187.5 m/min, $110.1 \pm$ 16.9 and $40.0-189.9 \mathrm{~m} / \mathrm{min}$, and $117.7 \pm 16.3$ and 74.6-168.5, respectively (trend among groups, $p<0.01$ ). The average and range of usual gait speed in the first, second, third, and fourth quartiles were 70.3 \pm 19.1 and 20.1-133.3, $81.3 \pm 16.4$ and 30.8-115.2, $87.1 \pm 14.3$ and 38.6-130.4,
Table 2 Correlation of speed of sound (SOS) with variables in the physical performance test

\begin{tabular}{ll}
\hline Variable & Value \\
\hline Weight bearing index & 0.038 \\
Grip strength (kg) & 0.055 \\
Single-leg balance with eyes closed (s) & 0.039 \\
Single-leg balance without eyes closed (s) & 0.035 \\
Maximum gait speed (m/min) & $0.111^{* *}$ \\
Step length (maximum speed gait) (cm) & $0.072^{*}$ \\
Usual gait speed (m/min) & $0.067^{*}$ \\
Step length (usual speed gait) (cm) & 0.029
\end{tabular}

Values are partial correlation coefficients adjusted for age and body mass index (BMI)

$* p<0.05, * * p<0.01$

and $93.0 \pm 14.2$ and 44.0-136.4 m/min, respectively (trend among groups, $p<0.01)$. We also found an association between gait speed and SOS in the four groups based on the age range: a significant association was found between maximum gait speed and SOS in all age groups except the oldest one.

The proportion of subjects with a usual gait speed of less than $48 \mathrm{~m} / \mathrm{min}$, the proposed cut-off value for sarcopenia screening [4], decreased as leg strength increased across all WBI quartiles, from $12.9 \%$ in the first quartile, to $3.7 \%$ in the second quartile, and $0.4 \%$ in the third and fourth quartiles.

Prediction of decreased bone strength by gait speed

Kishimoto et al. [16] determined the cut-off value for suspected osteoporosis or osteopenia among Japanese 
Table 3 Association between maximal or usual gait speed and SOS

\begin{tabular}{|c|c|c|c|c|c|c|c|c|c|}
\hline \multirow[t]{2}{*}{ Gait speed } & \multirow{2}{*}{$\begin{array}{l}\text { All } \\
(n=1,061)\end{array}$} & \multicolumn{4}{|c|}{ Weight bearing index } & \multicolumn{4}{|l|}{ Age } \\
\hline & & $\begin{array}{l}1 \mathrm{st} \\
(n=286)\end{array}$ & $\begin{array}{l}2 \text { nd } \\
(n=245)\end{array}$ & $\begin{array}{l}3 \mathrm{rd} \\
(n=271)\end{array}$ & $\begin{array}{l}\text { 4th } \\
(n=259)\end{array}$ & $\begin{array}{l}55-64 \\
(n=372)\end{array}$ & $\begin{array}{l}65-74 \\
(n=466)\end{array}$ & $\begin{array}{l}75-84 \\
(n=210)\end{array}$ & $\begin{array}{l}85-90 \\
(n=13)\end{array}$ \\
\hline \multicolumn{10}{|c|}{ Maximal gait speed } \\
\hline Unadjusted & $0.302 * *$ & $0.353 * *$ & $0.320 * *$ & $0.151 *$ & $0.187 * *$ & $0.118^{*}$ & $0.132 * *$ & $0.195 * *$ & -0.176 \\
\hline Adjusted $^{\mathrm{a}}$ & $0.111 * *$ & $0.163 * *$ & $0.148^{*}$ & 0.025 & 0.077 & 0.102 & $0.109^{*}$ & $0.170^{*}$ & -0.220 \\
\hline \multicolumn{10}{|l|}{ Usual gait speed } \\
\hline Unadjusted & $0.250^{*}$ & $0.326 * *$ & $0.311^{*}$ & 0.032 & 0.096 & 0.050 & $0.091 *$ & $0.161 *$ & -0.026 \\
\hline Adjusted $^{\mathrm{a}}$ & $0.067 *$ & $0.144 *$ & $0.150^{*}$ & -0.067 & 0.006 & 0.057 & 0.068 & 0.133 & -0.107 \\
\hline
\end{tabular}

Values are presented as partial correlation coefficients

$* p<0.05, * * p<0.01$

a Adjusted for age and BMI

women aged $20-89$ to be $1,479 \mathrm{~m} / \mathrm{s}$ for osteoporosis and $1,501 \mathrm{~m} / \mathrm{s}$ for osteopenia. Decreased bone strength was defined as an SOS level lower than $1,501 \mathrm{~m} / \mathrm{s}$. We found that the subjects in our study meeting these criteria were present across a broad range of gait speeds, namely, 79.4, $71.4,69.7$, and $67.2 \%$ of subjects in the first to fourth quartile, respectively, based on WBI. Likewise, 43.4, 34.3, 25.8 , and $26.6 \%$ of subjects in the first to fourth quartile, respectively, had an SOS level of less than $1,479 \mathrm{~m} / \mathrm{s}$.

Finally, receiver operating characteristic (ROC) curves for detecting persons with an SOS value of less than 1,501 or $1,479 \mathrm{~m} / \mathrm{s}$ revealed better test performance using maximum gait speed (Fig. 1). Since the SOS of most of the subjects aged 85 years and older is less than $1,479 \mathrm{~m} / \mathrm{s}$, this group of subjects was excluded from this analysis. The sensitivity and specificity of predicting individuals with a SOS of less than 1,501 or $1,479 \mathrm{~m} / \mathrm{s}$ showed a relatively high specificity among those with a low maximum gait speed (Fig. 2).

\section{Discussion}

The functional decline accompanied by aging has a critical impact on the quality of life of elderly persons. Changes in both the quantity and quality of muscle and bone play important roles in this process. Physical performance tests in geriatric medicine include gait speed, grip strength, and one leg standing tests $[17,18]$. Muscle strength and performance measurements are relatively easy to measure and are a potentially useful tool to evaluate functional decline and health status. These measurements are rarely done in clinical practice, however, partly because they do not directly diagnose diseases. Performance measurements (specifically gait) have been proposed as a diagnosis for sarcopenia [9]. In addition, gait speed has been associated with survival in older adults [7]. In the present study, we tested whether muscle strength or performance measurements were able to predict bone strength in a set of postmenopausal Japanese women and thus be useful as a screening tool for osteopenia or osteoporosis. Our data suggest that maximal gait speed, in combination with WBI, may be a useful and specific test to predict bone status.

Although gait speed has been shown to be influenced by multiple physiological and psychological factors [19], it is a usable test in clinical practice and has been shown to be an important predictor of health events. In our study, we examined whether gait speed predicts SOS values. Calcaneus measurements based on ultrasound and dual-energy X-ray absorptiometry (DXA) have been found to be useful tools for evaluating bone status $[20,21]$. The relationship between gait parameters and bone status has been examined using ultrasound parameters, and gait speed has been shown to be significantly associated with better bone quality in a cross-sectional study [22-24]. A significantly greater decline in usual gait speeds over a 2-year period in elderly women whose BMD decreased compared with those whose BMD increased has also been reported [25]. Sun et al. [23], showed that maximum gait speed had the strongest association with the stiffness index (SI) in youngold women and that daily walking steps (steps/day) had the strongest association with SI in old-old women. Maximum gait speed demands stronger limb-muscle strength and greater balance and declines relatively steeply with age $[25,26]$; thus, maximum gait speed tends to represent a higher mobility capability and to exert more effect on bone status than usual walking speed. Taken together, both maximum and usual gait speed can be a predictor of bone status, but maximum gait speed may have a relatively better correlation with bone status, as also suggested by our results.

We hypothesized that the association of gait speed and bone status might be different as leg strength changes. The association between leg strength and gait speed has been 

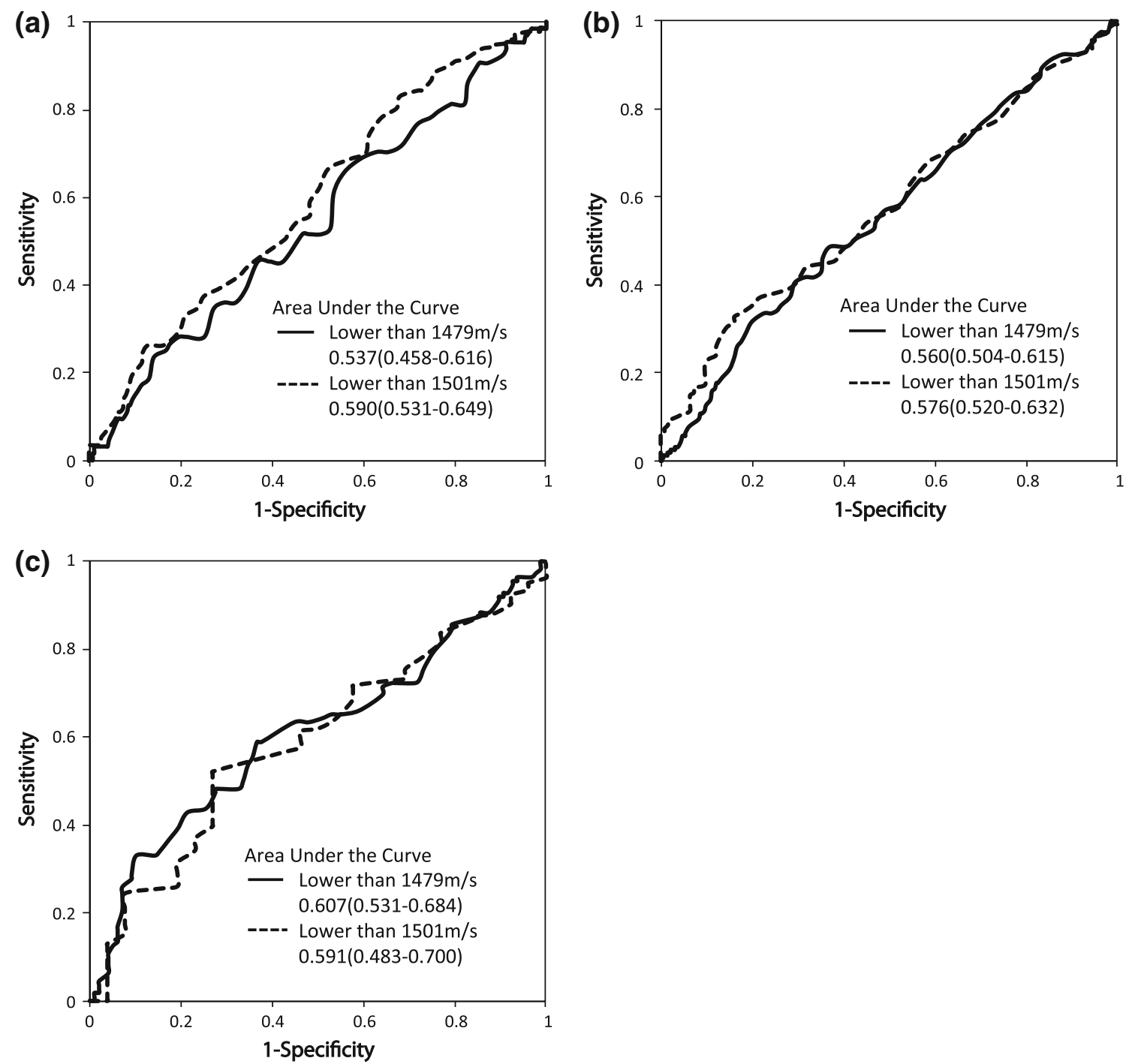

Fig. 1 Receiver operating characteristic analysis of maximum gait speed to detect a speed of sound (SOS) lower than 1,479 or 1,501 m/s in different age groups. a 55-64 years, b 65-74 years, c 75-84 years

shown to be non-linear and also to be present in weaker subjects but not in stronger ones [27]. Accordingly, we classified subjects into quartiles based on leg strength and analyzed the association. There were a considerable number of subjects with potential sarcopenia based on usual gait speed (i.e, gait speed $\leq 48 \mathrm{~m} / \mathrm{min}$ ) [9] in the first and second quartiles. Both maximal and usual gait speed had significant associations with SOS levels after adjustment for age and BMI within the quartiles. Therefore, the role of gait speed as a predictor for bone status may exist, especially among subjects with a lower level of muscle strength.

The significance of gait speed as a predictor of bone status has its highest specificity when the cut-off point of gait speed is set low, although sensitivity is very low under such conditions. Many individuals with a normal or high gait speed have low bone strength; however, subjects with very low gait speed are likely to be osteopenic. Obviously, there are multiple determinants for bone status, and it is difficult to rule out the possibility of impaired bone status based on gait. However, it is possible to predict bone status when walking speed is substantially decreased.

This study has several limitations. First, because it is a cross-sectional study, we are unable to discuss the significance of gait speed on an individual basis. Second, SOS measurement at the heel is directly affected by gait and might not reflect bone strength at other sites. Finally, it is important to note that decreased gait speed can predict not only bone status, but also the incidence of falls or bone fractures. In our study, both maximum and usual gait speed were significantly associated with single-leg balance with eyes open. When the eyes were closed, only maximum gait speed had a significant association with single-leg balance (data not shown). 
(a)

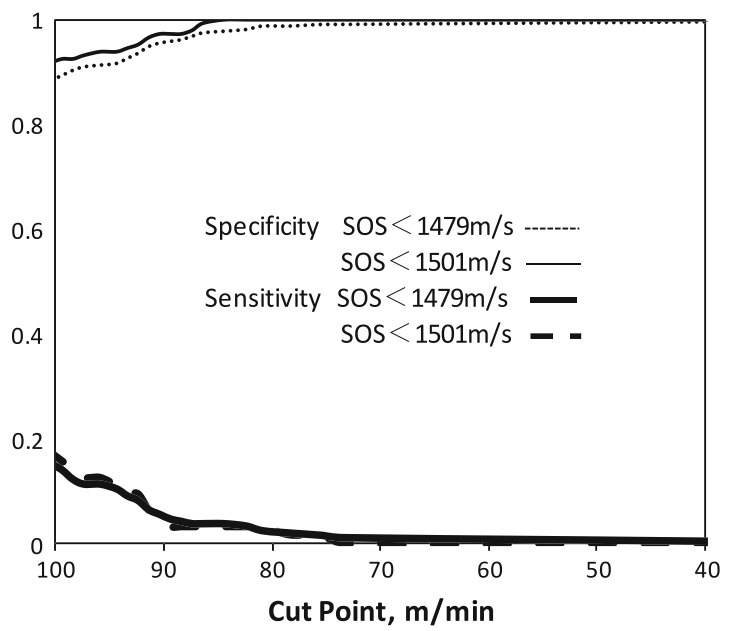

(c)

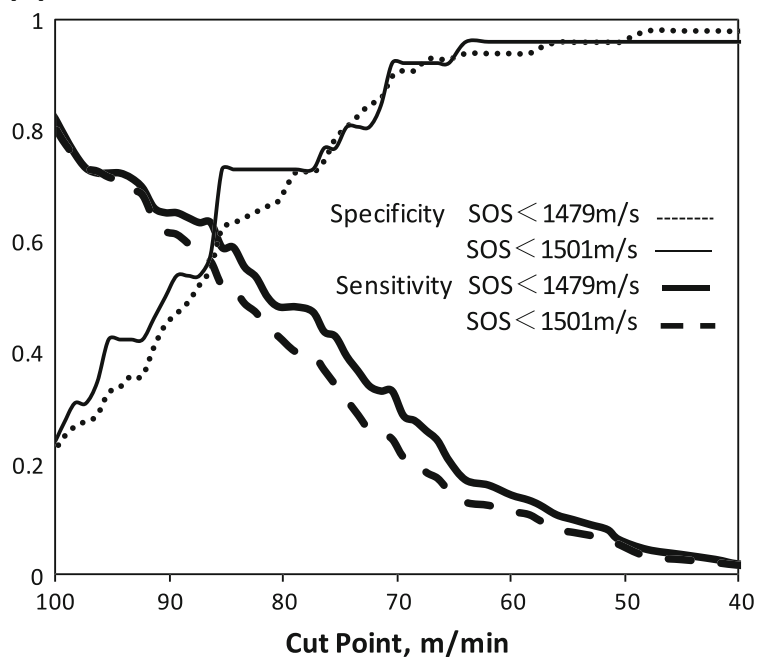

(b)

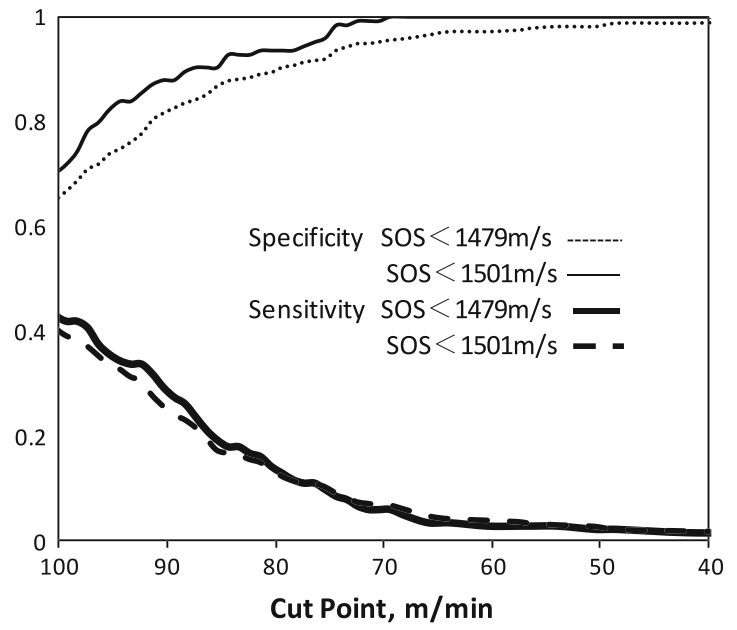

Fig. 2 Sensitivity and specificity for detecting a SOS lower than 1,479 or 1,501 m/s by maximum gait speed measurement in different age groups. a 55-64 years, b 65-74 years, c 75-84 years

In conclusion, the combination of WBI and gait speed is a specific method by which to screen subjects with low SOS values and, therefore, at high risk of bone fracture. It is currently not easy to clinically discriminate those individuals at high risk for critical fractures; therefore, gait speed and physical examination may become useful tools in clinical practice.

Acknowledgments This study was supported by the Wellness Core 21 nonprofit organization, Kobe, Japan.

\section{Conflict of interest None.}

\section{References}

1. Inouye SK, Studenski S, Tinetti ME, Kuchel GA. Geriatric syndromes: clinical, research, and policy implications of a core geriatric concept. J Am Geriatr Soc. 2007;55:780-91.
2. Waters DL, Baumgartner RN, Garry PJ. Sarcopenia: current perspectives. J Nutr Health Aging. 2000;4:133-9.

3. Raisz LG. Screening for osteoporosis. N Engl J Med. 2005;353: 164-71.

4. Cesari M, Kritchevsky SB, Newman AB, Simonsick EM, Harris TB, Penninx BW, et al. Added value of physical performance measures in predicting adverse health-related events: results from the Health, Aging and Body Composition Study. J Am Geriatr Soc. 2009;57:251-9.

5. Wennie Huang WN, Perera S, VanSwearingen J, Studenski S. Performance measures predict onset of activity of daily living difficulty in community-dwelling older adults. J Am Geriatr Soc. 2010;58:844-52.

6. Cooper R, Kuh D, Hardy R; Mortality Review Group; FALCon and HALCyon Study Teams. Objectively measured physical capability levels and mortality: systematic review and metaanalysis. Br Med J. 2010;341:c4467.

7. Studenski S, Perera S, Patel K, Rosano C, Faulkner K, Inzitari M, et al. Gait speed and survival in older adults. JAMA. 2011;305:50-8.

8. Dumurgier J, Elbaz A, Ducimetière P, Tavernier B, Alpérovitch A, Tzourio C. Slow walking speed and cardiovascular death in 
well-functioning older adults: prospective cohort study. Br Med J. 2009;339:b4460.

9. Cruz-Jentoft AJ, Baeyens JP, Bauer JM, Boirie Y, Cederholm T, Landi F, et al. Sarcopenia: European consensus on definition and diagnosis: report of the European Working Group on Sarcopenia in Older People. Age Ageing. 2010;39:412-23.

10. Hans D, Dargent-Molina P, Schott AM, Sebert JL, Cormier C, Kotzki PO, et al., for the EPIDOS Prospective Study Group. Ultrasonographic heel measurements to predict hip fracture in elderly women: the EPIDOS prospective study. Lancet 1996;348: 511-14.

11. Fujiwara S, Sone T, Yamazaki K, Yoshimura N, Nakatsuka K, Masunari $\mathrm{N}$, et al. Heel bone ultrasound predicts non-spine fracture in Japanese men and women. Osteoporos Int. 2005;16: 2107-12.

12. Schott AM, Hans D, Sornay-Rendu E, Delmas PD, Meunier PJ. Ultrasound measurements on os calcis: precision and age-related changes in a normal female population. Osteoporos Int. 1993;3: 249-54.

13. Judge JO, Underwood M, Gennosa T. Exercise to improve gait velocity in older persons. Arch Phys Med Rehabil. 1993;74: 400-6.

14. Yanagimoto Y, Takeda M, Hide K, You K. Relationships between bone strength in the calcaneus and the physical fitness or leg strength in the aged women (in Japanese). Osteopor Jpn. 2005;13:773-81.

15. Miyatake N, Takanami S, Kawasaki Y, Fujii M. Relationship between visceral fat accumulation and physical fitness in Japanese women. Diabetes Res Clin Pract. 2004;64:173-9.

16. Kishimoto H, Yoh K, Ohta H, Gorai I, Hashimoto J, Nakatsuka $\mathrm{K}$, et al. Reference and cut-off value of quantitative ultrasound measured by CM-100 (in Japanese). Osteoporos Jpn. 2003;11: 307-10.

17. Bohannon RW. Comfortable and maximum walking speed of adults aged 20-79 years: reference values and determinants. Age Ageing. 1997;26:15-9.

18. Shinkai S, Watanabe S, Kumagai S, Fujiwara Y, Amano H, Yoshida $\mathrm{H}$, et al. Walking speed as a good predictor for the onset of functional dependence in a Japanese rural community population. Age Ageing. 2000;29:441-6.

19. Tiedemann A, Sherrington C, Lord SR. Physiological and psychological predictors of walking speed in older communitydwelling people. Gerontology. 2005;51:390-5.

20. He YQ, Fan B, Hans D, Li J, Wu CY, Njeh CF, et al. Assessment of a new quantitative ultrasound calcaneus measurement: precision and discrimination of hip fractures in elderly women compared with dual X-ray absorptiometry. Osteoporos Int. 2000;11: 354-60.

21. Moris M, Peretz A, Tjeka R, Negaban N, Wouters M, Bergmann P. Quantitative ultrasound bone measurements: normal values and comparison with bone mineral density by dual X-ray absorptiometry. Calcif Tissue Int. 1995;57:6-10.

22. Sun W, Watanabe M, Tanimoto Y, Shibutani T, Saito M, Usuda $\mathrm{K}$, et al. Ultrasound parameters of bone health and related physical measurement indicators for the community-dwelling elderly in Japan. Geriatr Gerontol Int. 2007;7:154-9.

23. Sun W, Watanabe M, Tanimoto Y, Kono R, Saito M, Hirota C, et al. Assessment of the best gait parameter in relation to bone status in community-dwelling young-old and old-old women in Japan. Arch Gerontol Geriatr. 2009;49:158-61.

24. Yanagimoto Y, Oshida Y, Sato Y. Effects of walking on bone quality as determined by ultrasound in the elderly. Scand J Med Sci Sports. 2000;10:103-8.

25. Kwon J, Suzuki T, Yoshida H, Kim H, Yoshida Y, Iwasa H, et al. Association between change in bone mineral density and decline in usual walking speed in elderly community-dwelling Japanese women during 2 years of follow-up. J Am Geriatr Soc. 2007;55: $240-4$.

26. Malatesta D, Simar D, Dauvilliers Y, Candau R, Borrani F, Prefaut $\mathrm{C}$, et al. Energy cost of walking and gait instability in healthy 65- and 80-yr-olds. J Appl Physiol. 2003;95:2248-56.

27. Buchner DM, Larson EB, Wagner EH, Koepsell TD, de Lateur BJ. Evidence for a non-linear relationship between leg strength and gait speed. Age Ageing. 1996;25:386-91. 